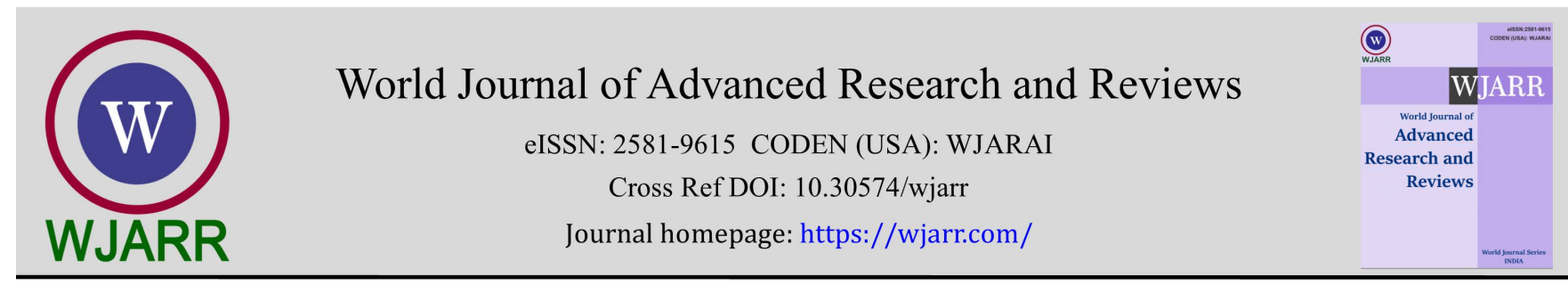

(RESEARCH ARTicle)

\title{
Kawasaki disease with low C-reactive protein levels
}

\author{
Toshimasa Nakada * \\ Department of Pediatrics, Aomori Prefectural Central Hospital, 030-8553 Higashi- tukurimiti 2-1-1, Aomori City, Aomori \\ Prefecture, Japan.
}

World Journal of Advanced Research and Reviews, 2021, 10(02), 241-245

Publication history: Received on 27 March 2021; revised on 30 April 2021; accepted on 03 May 2021

Article DOI: https://doi.org/10.30574/wjarr.2021.10.2.0191

\begin{abstract}
Kawasaki disease (KD) is an acute febrile systemic vasculitis that primarily affects children, and coronary artery lesions (CALs) are severe complications. Clinical features and outcomes of patients with KD associated with low serum Creactive protein (CRP) levels $(<3.0 \mathrm{mg} / \mathrm{dL})$ before initial treatment remain unclear. The objective of this retrospective study was to elucidate the clinical features and outcomes of patients with KD and low serum CRP levels. Data were included from 220 patients with KD who underwent acute-phase treatment from January 2009 to February 2021 in our department. Patients were divided into low group $(n=50)$ and high group $(n=170)$ according to serum CRP levels before initial treatment. The rates of incomplete KD (48.0\% vs. 10.6\%, P < 0.001) and serum albumin levels ( $\mathrm{g} / \mathrm{dL}$ ) before initial treatment (median 3.60 vs. 3.30, $\mathrm{P}<0.001$ ) were significantly different between the groups. The rate of patients who required intravenous immunoglobulin (IVIG) therapy was significantly lower in the low group compared to the high group $(74.0 \%$ vs. $97.1 \%, \mathrm{P}<0.001)$. However, the rates of initial IVIG therapy resistance $(10.8 \%$ vs. $25.5 \%$, $\mathrm{P}=0.055)$ and CALs (2.0\% vs. $4.7 \%, \mathrm{P}=0.475)$ were similar between the groups. No patient in the low group experienced CALs one month after KD onset. The severity of the disease in patients with KD and low CRP levels was milder than in those with high CRP levels.
\end{abstract}

Keywords: Coronary artery lesions; C-reactive protein; Intravenous immunoglobulin therapy; Kawasaki disease; Outcomes.

\section{Introduction}

Kawasaki disease (KD) is an acute febrile systemic vasculitis that primarily affects children younger than 5 years [1]. Coronary artery lesions (CALs) are severe complications of KD. Elevation of serum C-reactive protein (CRP) levels reflects systemic inflammation in KD [2]. In general, high serum CRP levels are expected in KD [2]. Therefore, in patients presenting with incomplete KD that does not fulfill the diagnostic criteria, a CRP serum level > $3.0 \mathrm{mg} / \mathrm{dL}$ is used as a criterion to confirm KD [3]. The high serum CRP levels is an index that predicts immunoglobulin-resistant KD and CAL development [4-6]. However, one study showed that CAL occurred in patients with both high and low CRP levels [2].

Current real-world clinical features and outcomes of patients with KD associated with low CRP levels $(<3.0 \mathrm{mg} / \mathrm{dL})$ before initial treatment remain unclear. Therefore, this retrospective study was conducted to elucidate the clinical features and outcomes of patients with KD and low serum CRP levels.

\footnotetext{
* Corresponding author: Toshimasa Nakada; Email: toshimasanakada@yahoo.co.jp

Department of Pediatrics, Aomori Prefectural Central Hospital ₹ 030-8553 Higashi- tukurimiti 2-1-1, Aomori City, Aomori Prefecture, Japan.
} 


\section{Material and methods}

\subsection{Participants and methods}

This study included data from 220 patients with KD who underwent acute-phase treatment from January 2009 to February 2021. Patients with a first episode of KD were included. Patients were divided into low group ( $\mathrm{n}=50$ ) and high group $(n=170)$ according to serum CRP levels before initial treatment.

KD was diagnosed based on the criteria (Japanese, fifth edition) mentioned in the diagnostic guidelines for KD until August 2019 and on the revised criteria (Japanese, sixth edition) from September 2019 [7, 8]. The Egami score, a risk score for predicting intravenous immunoglobulin (IVIG) resistance based on clinical findings such as age, days of illness, platelet count, alanine aminotransferase level, and CRP, was evaluated before the initial IVIG therapy [5].

\subsection{Initial Therapy}

During the study period, a single IVIG infusion of $2 \mathrm{~g} / \mathrm{kg} / \mathrm{dose}$ was given as initial therapy starting on day five of the illness, whenever possible [9]. Patients without inflammation or complications at presentation received acute-phase therapy without IVIG [9]. Between January 2009 and November 2017, anti-inflammatory drugs (aspirin or flurbiprofen) were initiated within $24 \mathrm{hr}$ after the initial IVIG infusion ended [10]. Aspirin and flurbiprofen were started at 30 and $3-5 \mathrm{mg} / \mathrm{kg} /$ day, respectively, then decreased to 5-10 and $3 \mathrm{mg} / \mathrm{kg} /$ day, respectively, once the patient became afebrile [10]. Each treating physician choose aspirin or flurbiprofen after considering the patient's liver function and the risk of Reye's syndrome during influenza season. After December 2017, low-dose aspirin (5 mg/kg/day) was commenced on days 8-10 of illness after completion of IVIG infusion, including the second course of therapy [9].

\subsection{Rescue Therapy}

The decision to use rescue therapies in resistant patients was made 48 to $72 \mathrm{hr}$ after completion of the initial IVIG infusion. Physicians made this decision using comprehensive clinical parameters, including body temperature, major KD symptoms, general condition, and laboratory data [9]. Course two of therapy comprised rescue IVIG infusion at 2 $\mathrm{g} / \mathrm{kg} /$ dose and course three comprised of an ulinastatin infusion, third course of IVIG therapy, or plasma exchange [9].

\subsection{Diagnosis of CAL}

Echocardiography was used to diagnose CALs based on the Japanese criteria reported by Kobayashi et al. [4]. A CAL was diagnosed if any examination showed an internal lumen diameter of $\geq 3 \mathrm{~mm}$ in a patient younger than 5 years or a diameter of $\geq 4 \mathrm{~mm}$ in a patient older than 5 years if the internal diameter of a segment was at least 1.5 times that of an adjacent segment or if the lumen appeared irregular. A transient CAL was defined as the disappearance of a CAL within 30 days of the illness.

\subsection{Statistical Analysis}

Statistical analyses were conducted using Stat Flex Version 6 for Windows (Artech Co., Ltd., Osaka, Japan). Chi-square, Fisher exact, and Mann-Whitney U tests were used as appropriate, with sample size considerations. $\mathrm{P}<0.05$ was considered statistically significant.

\section{Results and discussion}

The rates of male gender and age at KD onset between the low and high groups were similar (Table 1). The rate of incomplete KD of the low group was significantly higher than that of the high group (Table 1). The Egami score of the low group was significantly lower than that of the high group (Table 1). Furthermore, the serum albumin levels of the low group were significantly higher than those of the high group (Table 1). The day of illness of evaluation for albumin and CRP levels of the low group was significantly lower than that of the high group, whereas the median day of illness was 5.0 in the two groups (Table 1).

A total of 202 patients (91.8\%) received IVIG therapy, and 198 patients $(90.0 \%)$ underwent IVIG with delayed use of aspirin/flurbiprofen. The rate of patients who required IVIG therapy in the low group was significantly lower than that in the high group (Table 1). However, the rate of initial IVIG therapy resistance and CALs were similar between the groups (Table 1). No patient in the low group experienced CALs one month after KD onset (Table 1). 
Table 1 Comparison of basic characteristics, treatments, and outcomes between the low group and the high group

\begin{tabular}{|c|c|c|c|}
\hline Variables & Low group $(n=50)$ & High group $(n=170)$ & P-value \\
\hline Male gender & $28(56.0 \%)$ & $82(48.2 \%)$ & 0.334 \\
\hline Age at KD onset (months) & $19.5(13.0-35.0)$ & $28.0(16.0-49.0)$ & 0.070 \\
\hline Incomplete KD & $24(48.0 \%)$ & $18(10.6 \%)$ & $<0.001$ \\
\hline Egami score & $1.0(0.0-1.0)$ & $2.0(1.0-2.0)$ & $<0.001$ \\
\hline CRP (mg/dL) & $1.245(0.530-2.580)$ & $7.260(5.200-11.800)$ & $<0.001$ \\
\hline Albumin (g/dL) & $\begin{array}{l}3.60(3.40-3.85) \\
(n=48)\end{array}$ & $3.30(3.10-3.60)$ & $<0.001$ \\
\hline $\begin{array}{l}\text { Day of illness of evaluation } \\
\text { [mean+/- standard deviation] }\end{array}$ & $\begin{array}{l}5.0(5.0-6.0) \\
{[6.00+/-1.96]}\end{array}$ & $\begin{array}{l}5.0(5.0-6.0) \\
{[5.27+/-1.41]}\end{array}$ & 0.018 \\
\hline IVIG therapy & $37(74.0 \%)$ & $165(97.1 \%)$ & $<0.001$ \\
\hline $2 \mathrm{~g} / \mathrm{kg}$ IVIG therapy & $\begin{array}{l}36(97.3 \%) \\
(n=37)\end{array}$ & $\begin{array}{l}163(98.8 \%) \\
(n=165)\end{array}$ & 1.000 \\
\hline $\begin{array}{l}\text { Start day of illness of initial IVIG } \\
\text { therapy }\end{array}$ & $\begin{array}{l}5.0(5.0-6.0) \\
(n=37)\end{array}$ & $\begin{array}{l}5.0(5.0-6.0) \\
(n=165)\end{array}$ & 0.586 \\
\hline Non-responder & $\begin{array}{l}4(10.8 \%) \\
(n=37)\end{array}$ & $\begin{array}{l}42(25.5 \%) \\
(n=165)\end{array}$ & 0.055 \\
\hline $\begin{array}{l}\text { Low-dose aspirin/ } \\
\text { Medium-dose aspirin/ } \\
\text { Flurbiprofen }\end{array}$ & $\begin{array}{l}10(20.0 \%) \\
26(52.0 \%) \\
14(28.0 \%)\end{array}$ & $\begin{array}{l}37(21.8 \%) \\
80(47.1 \%) \\
53(31.2 \%)\end{array}$ & $\begin{array}{l}0.789 \\
0.539 \\
0.668\end{array}$ \\
\hline \multicolumn{4}{|l|}{ CAL } \\
\hline $\begin{array}{l}\text { Less than one month after } \mathrm{KD} \\
\text { onset }\end{array}$ & $1(2.0 \%)$ & $8(4.7 \%)$ & 0.475 \\
\hline One month after KD onset & $0(0.0 \%)$ & $4(2.4 \%)$ & 0.577 \\
\hline
\end{tabular}

The main findings of this study included that the severity of the disease in patients with KD and low CRP levels was milder than that of those with high CRP levels. Also, patients with KD and low CRP levels experienced favorable outcomes.

The results regarding Egami score, serum albumin levels, and the rate of the patients who required IVIG therapy in the low group suggested that the severity of the disease in patients with KD and low CRP levels was milder than that of those with high CRP levels. A low serum albumin level indicates severe arteritis in the acute-phase of KD, and is considered a risk factor for IVIG resistance and CAL development [11,12]. The lower Egami score and higher serum albumin levels before initial therapy in the low group suggest patients with less severe KD in this group (Table 1). In fact, the rate of the patients who required IVIG therapy in the low group was significantly lower than that in the high group (Table 1). The higher serum albumin levels before initial therapy and the lower rate of patients who required IVIG therapy in the low group were consistent with the findings by An et al [2].

The rate of non-responder in patients with low CRP levels was significantly lower than that of those with high CRP levels in the study by An et al. (11.7\% vs. $20.1 \%$, P < 0.001) [2]. In the present study, the rate of non-responder in the low group was lower than that in the high group, whereas it was not statistically significant because of the small sample size $(10.8 \%$ vs. $25.5 \%, P=0.055)$. Those findings regarding non-responder also suggest that the severity of the disease in patients with KD and low CRP levels was milder compared to those with high CRP levels. 
CAL occurred in patients with both high and low CRP levels according to findings by An et al. and the present study [2]. However, the findings regarding the rate of CAL between patients with low CRP levels and those with high CRP levels were different in those studies [2]. The rate of CAL based on Japanese criteria for patients with low CRP levels was significantly higher than that for patients with high CRP levels, both in the acute $(12.5 \%$ vs. 9.9\%, P = 0.001) and convalescent phases (6.8\% vs. $4.6 \%, \mathrm{P}<0.001)$ according to the findings by An et al. [2]. On the other hand, the rate of CAL based on Japanese criteria for patients with low CRP levels was similar to that for those with high CRP levels, both less than one month after KD onset $(2.0 \%$ vs.4.7\%, $\mathrm{P}=0.475)$ and one month after KD onset $(0.0 \%$ vs. $2.4 \%, \mathrm{P}=0.577)$ in the present study. One of the reasons for this difference could be attributed to the different acute-phase treatments for KD.

IVIG therapy at $2 \mathrm{~g} / \mathrm{kg}$ with concomitant use of medium/high-dose aspirin is the mainstay of the current standard therapy for acute-phase KD [3]. The majority of patients with KD in Korea received this standard therapy according to the findings of the study based on the data of the nationwide survey of KD conducted in Korea [13]. The study by An et al. was also conducted based on the data of the nationwide survey of KD in Korea [2]. On the other hand, the majority of patients with KD (90.0\%) in the present study received IVIG therapy at $2 \mathrm{~g} / \mathrm{kg}$ with delayed use of aspirin/flurbiprofen. Studies indicate that IVIG therapy at $2 \mathrm{~g} / \mathrm{kg}$ with delayed use of aspirin/flurbiprofen leads to the favorable outcomes of CAL caused by KD $[9,10,12]$.

Delay of the diagnosis and initial treatment in patients with incomplete KD may be another reason for the high rate of CAL development in patients with low CRP level in the study by An et al. [2]. Incomplete presentation of KD is a potential risk factor for delay of diagnosis and initial treatment [14]. The rate of incomplete KD in patients with low CRP levels was significantly higher than that in patients with high CRP levels in the present study and that by An et al. [2]. Therefore, patients with KD and low CRP levels have the potential risk of delay in diagnosis and initial treatment, as well as CAL development. In fact, the day of illness of evaluation before treatment in the low group was significantly later than that of the high group in the present study (Table 1). The findings by An et al. showed that the fever duration before treatment in patients with low CRP levels was longer than that in those with high CRP levels, but not statistically significant [2].

The rate of the patients with low CRP levels in the present study was consistent with that by An et al (23\% in both studies) [2]. Although not statistically significant, the age at KD onset of the low group was younger than that of the high group (Table 1). In the study by An et al, the age at KD onset of patients with low CRP levels was significantly younger than that of those with high CRP levels [2]. Careful attention and early evaluation, using echocardiography, for diagnosis of KD in young patients with a few major signs of KD and with low CRP levels are important because CAL occurs in patients with low CRP levels, as well as in those with high CRP levels. The limitations of this study include the small sample size and the retrospective study design.

\section{Conclusion}

The severity of the disease in patients with KD and low CRP levels was milder than in those with high CRP levels. Also, outcomes of patients with KD and low CRP levels was favorable, whereas CAL occurred in both patients with high and low CRP levels.

\section{Compliance with ethical standards}

\section{Acknowledgments}

I would like to thank all of those who were involved in the medical management of the patients included in this study, and Enago (www.enago.jp) for the English language review.

\section{Disclosure of conflict of interest}

None.

\section{Statement of ethical approval}

Our institutional ethics committee approved the study protocol and waived the requirement of patient consent because of the retrospective nature of the study. 


\section{References}

[1] Kawasaki T, Kosaki F, Okawa S, Shigematsu I, Yanagawa H. A new infantile acute febrile mucocutaneous lymph node syndrome (MLNS) prevailing in Japan. Pediatrics. 1974; 54: 271-276.

[2] An HS, Kim GB, Song MK, Lee SY, Kwon HW, Lee JW, Bae EJ. The Occurrence of Coronary Artery Lesions in Kawasaki Disease Based on C-reactive Protein Levels: A Retrospective Cohort Study. Research Square DOI: https://doi.org/10.21203/rs.3.rs-113714/v1

[3] Mc Crindle BW, Rowley AH, Newburger JW, Burns JC, Bolger AF, Gewitz MG, et al. Diagnosis, treatment, and longterm management of Kawasaki Disease: a scientific statement for health professionals from the American Heart Association. Circulation. 2017; 135: 927-99.

[4] Kobayashi T, Inoue Y, Takeuchi K, Okada Y, Tamura K, Tomomasa T, et al. Prediction of intravenous immunoglobulin unresponsiveness in patients with Kawasaki disease. Circulation. 2006; 113: 2606-12.

[5] Egami K, Muta H, Ishii M, Suda K, Sugahara Y, Iemura M, et al. Prediction of resistance to intravenous immunoglobulin treatment in patients with Kawasaki disease, J Pediatr. 2006; 149: 237-40.

[6] Yan F, Pan B, Sun H, Tian J, Li M. Risk factors of coronary artery abnormality in children with Kawasaki disease: a systematic review and meta-analysis. Frontiers in Pediatrics. 2019; 7: 374.

[7] Ayusawa M, Sonobe T, Uemura S, Ogawa S, Nakamura Y, Kiyosawa N, et al. Revision of diagnostic guidelines for Kawasaki disease (the 5th revised edition). Pediatr Int. 2005; 47: 232-4.

[8] Fukazawa R, Kobayashi J, Ayusawa M, Hamada H, Miura M, Mitani Y, et al. JCS/JSCS 2020 Guideline on Diagnosis and Management of Cardiovascular Sequelae in Kawasaki Disease. Circ J. 2020; 84: 1348-1407.

[9] Nakada T. Acute Phase Treatment and Medium-Term Outcomes in Kawasaki Disease. European Journal of Medical and Health Sciences 2020; 2 (4), August: 1-7.

[10] Nakada T. Effects of anti-inflammatory drugs on intravenous immunoglobulin therapy in the acute phase of Kawasaki disease. Pediatr Cardiol. 2015; 36: 335-9.

[11] Liu MY, Liu HM, Wu CH, Chang CH, Huang GJ, Chen CA, et al. Risk factors and implications of progressive coronary dilatation in children with Kawasaki disease. BMC Pediatrics. 2017; 17: 139.

[12] Nakada T: Inhibitory Effect of Anti-Inflammatory Drugs on the Initial Intravenous Immunoglobulin Therapy (2 $\mathrm{g} / \mathrm{kg} /$ dose) in Kawasaki Disease. International Journal of Advances in Medical Sciences. 2018; 3 (5): 1-10.

[13] Kim GB, Yu JJ, Yoon KL, Jeong SI, Song YH, Han JW, et al. Medium- or Higher Acetylsalicylic Acid for Acute Kawasaki disease and Patient Outcomes. J Pediatr. 2017; 184: 125-9.

[14] Wilder MS, Palinkas LA, Kao AS, Bastian JF, Turner CL, Burns JC. Delayed diagnosis by physicians contributes to the development of coronary artery aneurysms in children with Kawasaki syndrome. Pediatr Infect Dis J. 2007; 26(3): 256-60. 